to an end, even if the body that supports that life is well and functioning normally. As Albert Camus the existentialist philosopher argued, suicide is the fundamental philosophical problem, since in thinking about suicide one affirms or denies one's existence. The lab at Harvard and the research endeavours contained therein served as a powerful symbol of the worthiness of life and the struggle to understand those who, in their darkest moment, end it all.
PANDORA'S BOX

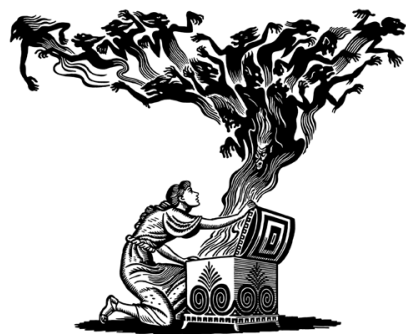

literature for evidence, news and other sources on matters of interest (doesn't shy away from controversy) to bring to the reader. She welcomes comments and suggestions (via ip@rcpsych. ac.uk)

\section{How suggestible are you?}

Tot long ago, memory of childhood trauma and in particular sexual abuse emerging in the context of recovered memory therapy caused a lot of debate among mental health professionals. It also had legal ramifications, as some cases were brought to court. The American Psychiatric Association and similar bodies worldwide condemned the 'recovered memory approach'. A Royal College of Psychiatrists' Working Group (Brandon et al) issued the following statement in 1998 :

No evidence exists for the repression and recovery of verified, severely traumatic events, and their role in symptom formation has yet to be proved. There is also striking absence in the literature of well-corroborated cases of such repressed memories recovered through psychotherapy. Given the prevalence of childhood sexual abuse, even if only a small proportion are repressed and only some of them are subsequently recovered, there should be a significant number of corroborated cases. In fact there are none. (See http://bjp.rcpsych.org/ content/172/4/296)

The subject has also attracted a lot of interest among neuroscientists. They have developed a technique called 'memory implantation' to investigate the 'false-memory' phenomenon. Suggestible individuals can be induced to believe an event happened in their childhood which is completely fallacious. There are various methods both of memory implantation, and of interpreting the degree to which participants have accepted the false memory as true. Some scientists who have used 'memory implantation' claim great success, others not, and this unreliability is likely to be due to non-standardised experimental techniques. Recently, a research group claimed to have developed a reliable coding system for 'false memories', which they applied to over 400 transcripts from eight peer-reviewed published 'memory implantation' studies. When the memory implantation technique provided idiosyncratic (personal) information to the participant, together with encouragement to imagine the (implanted) event, the false memory formation rate was $46.1 \%$. Surprisingly, providing false photographic 'evidence' worked against success.

If indeed we are so suggestible, how reliable can witnesses' evidence be in court?

Scoboria, A., Wade, K. A. D., Lindsay, S., et al (2016) A meta-

analysis of memory reports from eight peer-reviewed false memory implantation studies. Memory. https://doi.org/10.1080/09658211. 2016.1260747

\section{Why are political beliefs so fixed?}

Tave you wondered why some people's beliefs are not amenable to modification despite good evidence to the contrary? Researchers have shown that there is a good neurobiological basis to this! They used neuroimaging to investigate the neural basis of strongly held political beliefs in 40 people. During brain scanning, the participants were presented with political statements they strongly believed and then shown counter-claims that challenged each statement. Challenging their political beliefs increased activity in the default network, a set of interconnected structures which is thought to be concerned with high-level thinking about important personal beliefs or values, self-representation and disengagement from the external world. Greater belief-change resistance was associated with an increase in activity in the dorsomedial prefrontal cortex and a decrease in the orbitofrontal cortex. Those who were most resistant to changing their views showed more activity in the amygdala and the insula when evaluating the counter-evidence than did those more willing to change their mind. The amygdala is involved in the perception of threat and anxiety and the insular cortex detects the emotional salience of stimuli. These findings suggest that emotion plays a role in cognition, in reasoning and in belief-change resistance.

Kaplan, J. T., Gimbel, S. I. \& Harris, S. (2016) Neural correlates of maintaining one's political beliefs in the face of counterevidence. Scientific Reports. https://doi.org/10.1038/srep39589.

\section{When the heart weakens, the brain suffers}

Clinical signs of heart or brain damage emerge 4 some years after the onset of pathological changes and detecting these at an earlier stage may help prevent or delay the onset of such devastating conditions. Recent research findings from the Netherlands offer some hope of early detection. In 2397 people (mean age 57 years) without dementia, stroke or heart disease, drawn from the population-based Rotterdam Study, the authors examined the association between serum levels of $\mathrm{N}$-terminal pro-B-type natriuretic peptide (NT-proBNP), which is a marker of heart disease (cardiac wall stress), and magnetic resonance markers of subclinical brain damage (volumetric, focal and microstructural).

Higher concentrations of NT-proBNP were associated with smaller total brain volume, affecting 